\title{
ARTICLE
}

\section{Augmented Reality Based on Object Recognition for Piping System Maintenance}

\section{Ana Regina Mizrahy Cuperschmid* Mariana Higashi Sakamoto}

School of Civil Engineering, Architecture and Urban Design, Department of Architecture and Construction, University of Campinas (UNICAMP), Campinas, SP, 13083-889, Brazil

\begin{tabular}{l}
\hline ARTICLE INFO \\
\hline Article history \\
Received: 1 June 2021 \\
Accepted: 21 June 2021 \\
Published Online: 20 June 2021 \\
\hline Keywords: \\
Augmented reality \\
3D object tracking \\
Maintenance \\
Pipe \\
Facility management
\end{tabular}

\section{Introduction}

Augmented Reality (AR) is a technology that allows a composite visualization of the physical environment superimposed with virtual elements ${ }^{[1]}$. AR is a subdomain of Mixed Reality, defined as the intermediate environment between real and virtual worlds ${ }^{[2]}$. It is an alternative and complementary technology to traditional means of accessing information since it facilitates the data interpretation by overlapping virtual elements in the real world, considering the time, place and the real context of the design ${ }^{[3]}$.

AR can contribute to the Architecture, Engineering, Construction and Operation (AECO) processes as it can structure additional information that enables better

\section{ABSTRACT}

Augmented Reality (AR) applications can be used to improve tasks and mitigate errors during facilities operation and maintenance. This article presents an AR system for facility management using a three-dimensional (3D) object tracking method. Through spatial mapping, the object of interest, a pipe trap underneath a sink, is tracked and mixed onto the AR visualization. From that, the maintenance steps are transformed into visible and animated instructions. Although some tracking issues related to the component parts were observed, the designed AR application results demonstrated the potential to improve facility management tasks.

understanding. The complex nature of AECO makes the use of AR ideal on at least three levels: visualization, information retrieval and interaction ${ }^{[4]}$. This complexity is observed in the maintenance work of Mechanical, Electrical and Plumbing (MEP) systems. The difficulties that MEP operators face, range from the need for expertise in the area for interpreting MEP technical drawings loaded with symbology to the lack of updated information for asbuilt maintenance ${ }^{[5]}$.

Hence, AR can offer maintenance procedure visualization in more natural and interactive way because it can present, in real time, superimposed models and information on the physical world. The maintenance of plumbing systems supported by AR technology has great

*Corresponding Author:

Ana Regina Mizrahy Cuperschmid,

School of Civil Engineering, Architecture and Urban Design, Department of Architecture and Construction, University of Campinas (UNICAMP), Campinas, SP, 13083-889, Brazil;

Email: cuper@unicamp.br 
potential, because end users do not have the expertise and easy access to the maintenance data of systems like specialists. This study assumes that AR has the potential for aiding the maintenance of plumbing systems, as it presents a more intuitive and accessible communication for the non-specialist user.

The issue that leads this research is whether and how AR could be used as a tool to assist the maintenance of plumbing systems. The proposal is to convert the maintenance steps into visible and explicit visualization through an AR experience. To contextualize the research and allow the exploration, the maintenance process of a pipe trap underneath a sink is used. Among the main problems about this equipment are: odor entry into the environment, clogging, preventing the flow of sanitary sewage, and leakage, compromising the environment in which it is installed ${ }^{[6]}$.

\section{Related Works}

In the last five years, numerous studies have been conducted on the use of AR to assist operation and maintenance processes of pipe systems. AR has been applied to, e.g., examine its relation to human spatial cognitive abilities on assembly ${ }^{[7]}$; assist facility management using the image-based indoor localization ${ }^{[8]}$; improve work productivity through a piping management system ${ }^{[9]}$; locate virtual pipes behind a real wall with "X-ray vision" to establish relationships between task performance and spatial factors ${ }^{[10]}$; develop a framework to extract and visualize 3D models of structures connected with pipes ${ }^{[11]}$; understand spatial cognition on craftworker eye-gaze patterns in building a scale-model pipe assembly ${ }^{[12]}$; provide an application scenario for cooling tower and pipe shutdown protocol ${ }^{[5]}$. These related cases are summarized in Table 1.

Table 1. Related works

\begin{tabular}{ccc}
\hline & Description & Reference \\
\hline \multirow{4}{*}{ Objective } & $\begin{array}{c}\text { Framework development to test AR impact } \\
\text { for inspection or maintenance }\end{array}$ & {$[5,8,9,11]$} \\
& $\begin{array}{c}\text { Compare user performance in assembling } \\
\text { or locating tubes with different information }\end{array}$ & {$[7,10,12]$} \\
& formats & \\
& Quantitative and Statistical Analysis & {$[7,10,12]$} \\
Evaluation & The application of ETS cognitive tests & {$[7,9,12]$} \\
& Qualitative analysis - Performance & {$[5,8,9,11]$} \\
& evaluation & {$[9]$} \\
Tracking & marker based & {$[5,7,8]$} \\
& markless based & {$[8,12]$} \\
Display & HMD & {$[7,9]$} \\
device & Tablet & {$[5,11]$} \\
& Smartphones & {$[10]$} \\
\hline
\end{tabular}

These studies can be classified into two groups, according to their macro-goals: (i) system development for task optimization and (ii) human spatial cognition during operation and maintenance tasks.

Within the first macro-proposal there were cases of application development for the maintenance of leaks in a university building in Korea ${ }^{[8]}$ and in Taiwan ${ }^{[5]}$. Some cases were applied to piping systems of the naval industry for the control of tubes for shipbuilding ${ }^{[9]}$ and for the maintenance of offshore equipment ${ }^{[11]}$.

The cases that fall under the second macro-proposal involve performance studies: in the assembly of tubes aided by AR versus traditional paper based drawings [7]; for positioning tubes on the wall with the aid of AR ${ }^{[10]}$; and in the assembly of tubes measured using eyetracking technology ${ }^{[12]}$. In these cases, the assembling activities, when assisted by AR, presented better performance results - especially for people with little experience in the area or low spatial cognition ability ${ }^{[12]}$.

The technological solution adopted was approached in different ways by the authors. In all cases the integration of AR and Building Information Modeling (BIM) was mentioned, except the case involving the application for a ship factory ${ }^{[9]}$.

Overall, the studies discussed the disadvantages and advantages of four types of AR displays: Head Mounted Display (HMD), tablets, smartphones, and laptops. The HMDs were seen by maintenance professionals as impractical due to the size and inaccessible due to the price, in addition to representing a risk to the operator's safety ${ }^{[7]}$, while the notebook presented the setback of being fixed, which was mentioned to be a disadvantage for the notion of depth in the pipe assembly task ${ }^{[10]}$. However, smartphones and tablets have the advantage of being more portable and mobile, in addition, its builtin flashlight can illuminate dark indoor or outdoor night environments ${ }^{[5]}$.

An essential part of AR application is the correct connection between virtual information and the real world ${ }^{[13]}$. One way to connect this information is using markers, recognized in real-time and used as a reference object to precisely superimpose virtual 3D content onto the camera's live view. Markers were used in large quantities to identify tubes, and each tube received a unique identifying sticker that allowed access to information about the shipbuilding process ${ }^{[9]}$. The disadvantage of this technology is that there is a risk that the marker will be damaged by bad weather and that there is a need for prior installation.

Another way of connecting virtual data is through Simultaneous Localization and Mapping (SLAM) 
technology. This technology was used with the HMD Microsoft HoloLens ${ }^{[8]}$, and a smartphone ${ }^{[5]}$ and was applied together with a structured light scanner and an iPad ${ }^{[7]}$. This markless technology advantage is that it does not interfere with the environment aesthetics ${ }^{[1]}$.

Other similarities found in the related works involve the forms of assessment used in the studies. Most studies performed usability tests by inviting multiple participants. The studies that rated their participants' spatial cognitive abilities adopted ETS tests for 2D and 3D image recognition. Four studies performed a quantitative analysis of their results, statistically analyzing explanatory variables about the performance of the participants. While the other three cases applied a qualitative analysis, interviewing participants about feedback on the proposed solution.

\section{Methodology}

The proposed procedure, expressly addressed to the pipe trap maintenance aided by AR, consists of four main stages (i) establish the maintenance requirements; (ii) model the components; (iii) develop the AR application; (iv) evaluate the application performance.

\subsection{Maintenance Requirements}

The user experience level in relation to the piping system maintenance domain can influence the sink trap maintenance procedure. Therefore, users with little experience may present some difficulties, such as (i) recognition of the components, since each sink trap model or brand has its particularities; (ii) small parts, metal rings and sealing rubbers can easily get lost during the procedure or be misplaced in the fittings; (iii) the components assembly and reassembly requires a correct order, otherwise there is a risk of deforming the parts or leaking.

From a practical experimentation with the procedures that users normally make for the installation and maintenance of a cup-shaped trap, especially in the clogging event, it was possible to establish the potential contributions of the proposed system.

The procedure performed was (1) sink trap removal and cleaning; ( 2 and 3 ) sealing the two threaded pipe connections with PTFE tape; and (4) reassembling the trap and checking for leaks, Figure 1.
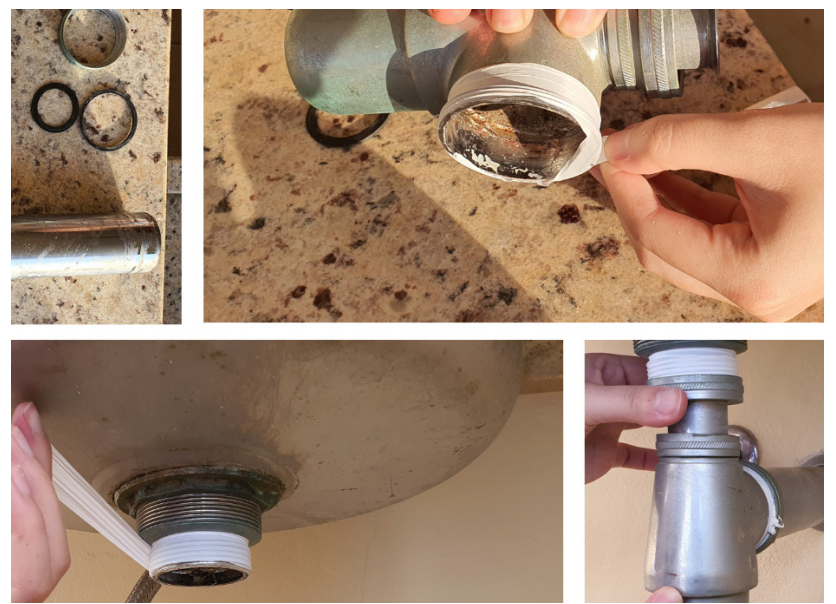

Figure 1. Sink trap maintenance process

Aiming to reduce the difficulties raised in the sink trap installation and maintenance procedure, five main requirements for applying the proposed AR application were defined: (1) list the sink trap components; (2) identify/recognize the main components; (3) warning of rubber seal's position; (4) order of components in the assembly; (5) present the image of the complete sink trap installed.

\subsection{Components Modeling}

Duratex, a Brazilian manufacturer, under the Deca e Hydra brands, produces valves for discharging the water systems of toilets, faucets for bathrooms and kitchens, among other related products. The Deca brand makes available on its website, a library of its products in different file formats for AutoCAD (DWG), Revit (RFA) and Sketchup (SKP). For this research, it is used for both physical and digital models of the sink trap.

To add details to the digital model, the Revit family RFA format file was used as the basis. The Revit software was chosen because it is a popular BIM software in the AECO industry. From the solid modeling tools, such as extrusion and revolution, the necessary details were added, such as the connection to the waste pipe, Figure 2.

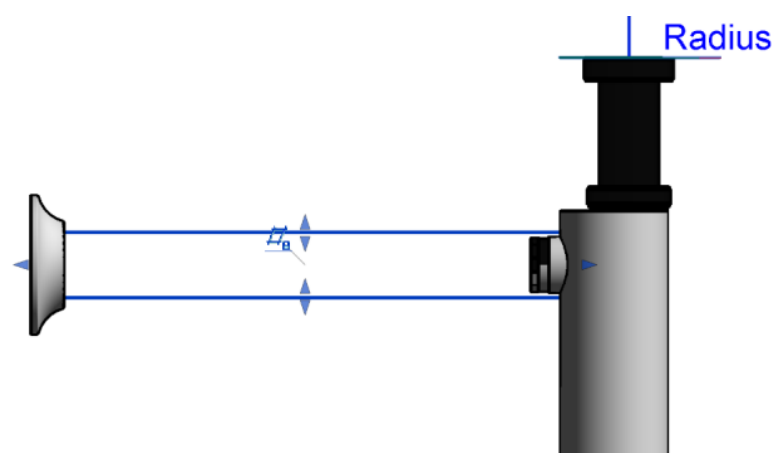

Figure 2. Sink trap modeling using Revit 
The sealing parts and the inspection cover were also modeled using the combination of elementary solids, in this case a cylinder and a torus and add different colors and textures from images. The addition of fitting elements and sink trap components was necessary to provide visual information about all parts, allowing the development of the maintenance steps.

\subsection{Application Development}

To develop an application with three-dimensional (3D) object recognition, it was necessary to choose an SDK. Several SDKs offer this possibility, as Vuforia ${ }^{[14]}$, Wikitude ${ }^{[15]}$ and VisionLib ${ }^{[16]}$. From the VisionLib SDK for Unity ${ }^{[17]}$, it is possible to create AR experiences for platforms compatible with Windows Desktop, iOS and Android, but the most interesting is the possibility of creating web based experiences.

Unity is an ecosystem for developing applications, games, or multi-platform 2D and 3D experiences. This engine was also the most cited, three of the seven related cases used this tool.

According to the VisionLib documentation, the potential of this technology lies in the fact that it improves computer vision through model-based tracking. The advantage of this tracking over SLAM tracking, is the greater accuracy for locating a point in the real world and with a higher level of stability, as it is not affected by changes in lighting or object movement.

It was decided to use VisionLib, because: (i) objectbased AR for the piping system maintenance was not yet identified in related cases; (ii) versatility in the creation of Unity's multi-platform interfaces and resources; (iii) it was possible to obtain a valid academic license during the development of this research.

Based on the requirements, an application was developed considering a sink trap exploded view, 3D object animation, and maintenance step visualization. First, it was decided to show an exploded view of the sink trap components, accompanied by an animation. The animation shows the complete set to its exploded view - aiding the visualization of each component in the recommended position.

The instructions (according to the manufacturer) were divided into four steps: (1) screwing the trap in the sink, (2) measuring distance between the tail pipe and the wall, (3) installing and (4) maintenance. In the application, each step was accompanied by a small animation to better illustrate the instruction.

The user interface was developed in Unity. It was composed of a panel containing the sink trap handling information and the tracked object visualization on the screen, accompanied by an animation. User interaction with AR application can happen through buttons, or by moving the camera at different angles or by clicking/ touching the screen - on the green cylindrical mesh attached to the model, Figure 3.

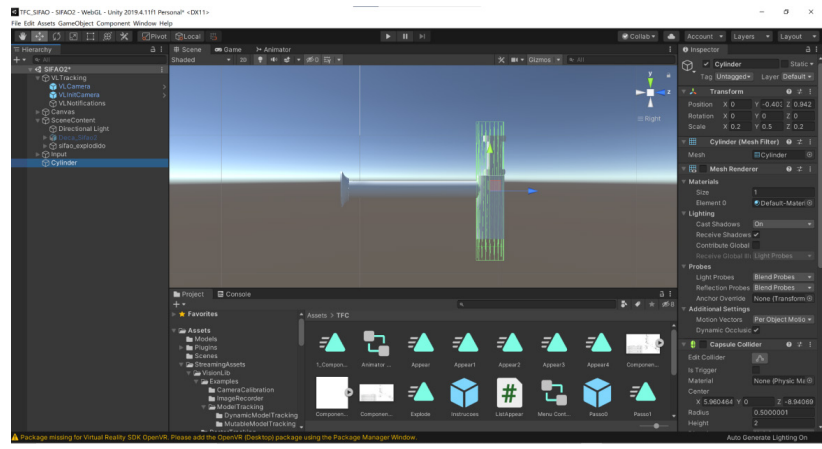

Figure 3. User interface development in Unity

The Unity left-side menu contains all the application objects, including VL Tracking (VisionLib pre-configured features), Canvas (panels containing the instructions textual elements) and SceneContent (composed of the scene objects). The right-side menu shows the selected element properties. The bottom menu presents a library or user objects that can be used in the application.

A C\# script was used to customize user interactions with objects in the scene. The Animator Controller was applied to order the animation, Figure 4. Each gray box represents an animation of the sink trap digital model.

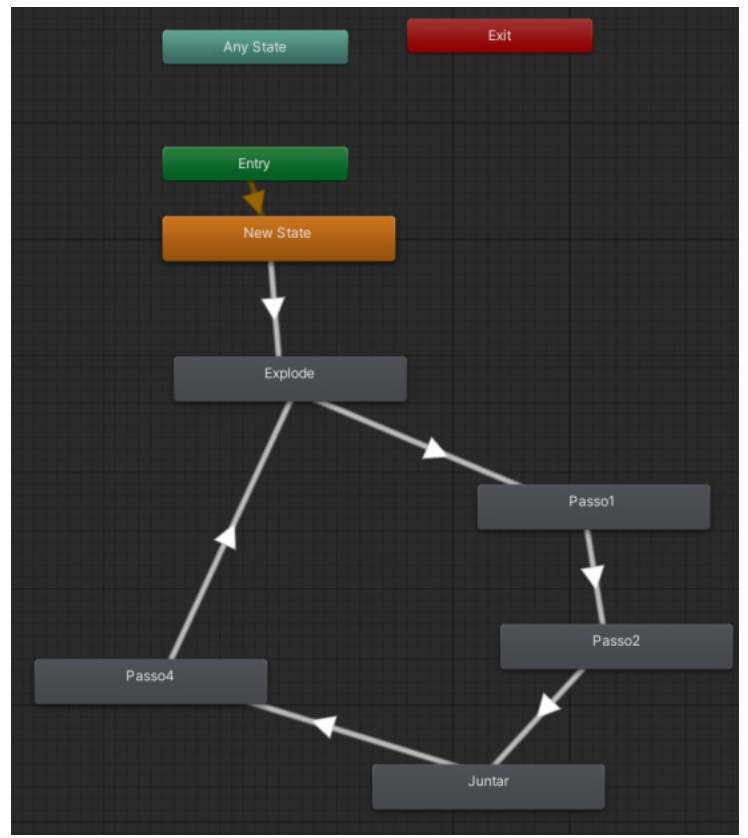

Figure 4. Animator Controller: animation order

Each animation was recorded separately. In a timeline, it was positioned frame by frame the objects on the scene, Figure 5. 


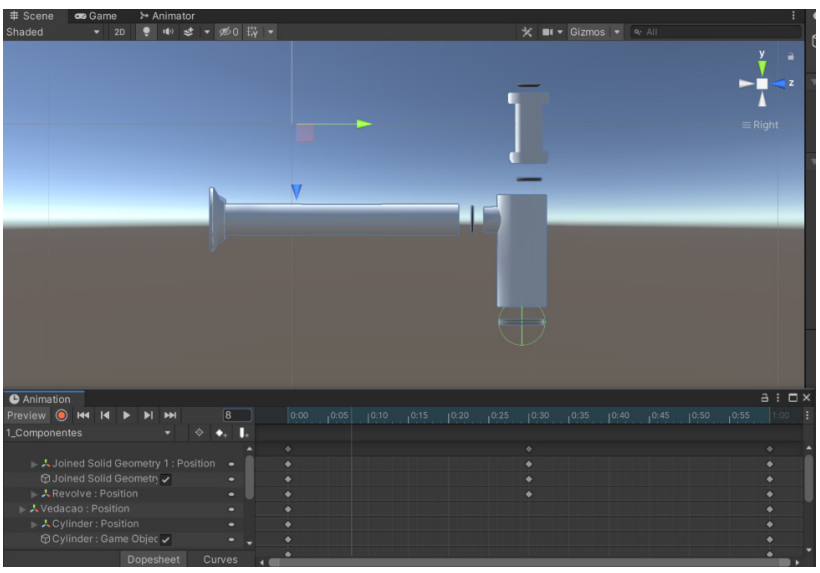

Figure 5. Animation development in Unity

\subsection{Evaluation}

In this section, the performance of the developed application is evaluated. The developed application tracks the physical sink trap to overlay maintenance information in $\mathrm{AR}^{1}$, Figure 6.

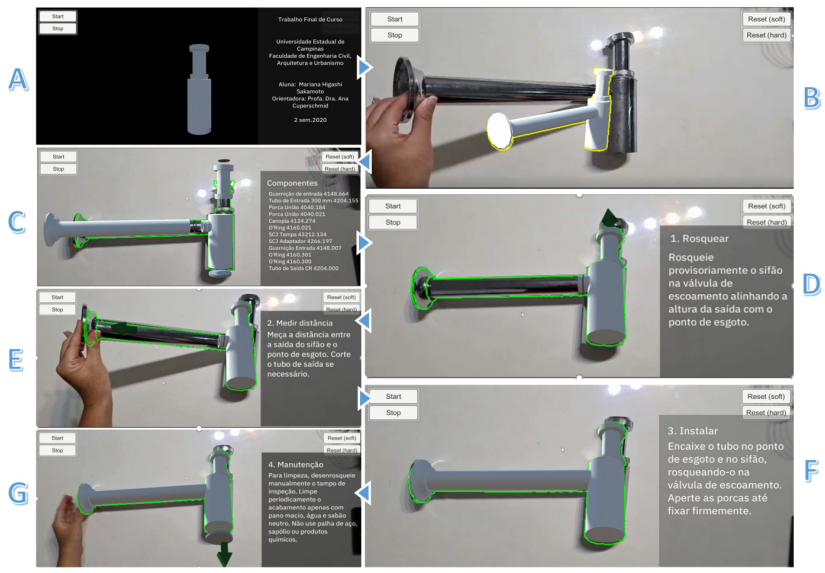

Figure 6. AR application evaluation

The initial screen (A) presents the application general information and its authorship. When pressing the start button, it is possible to choose which camera connected to the device will feed the AR.

Initially, the 3D model appears on the screen with a red outline and the Model Tracking feature is activated. When the geometry of the real sink trap is partially detected, the digital model outline changes to yellow according to (B). Then, when fully tracking the object, it changes its color to green and the digital model is superimposed on the real object. So, when approaching the camera or moving the real object, the digital model also accompanies the sink trap.

Scene (C) shows a list of the components for replacement and an animation of the exploded model in its

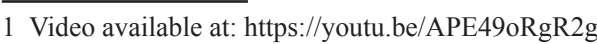

three demountable parts (trap, tail pipe and waste pipe) and other parts (sealing rubbers and inspection cover). When the camera approaches the real sink trap, it is possible to view in more detail the sealing rubbers evidenced by the scene (C) with an exploded view, Figure 7.

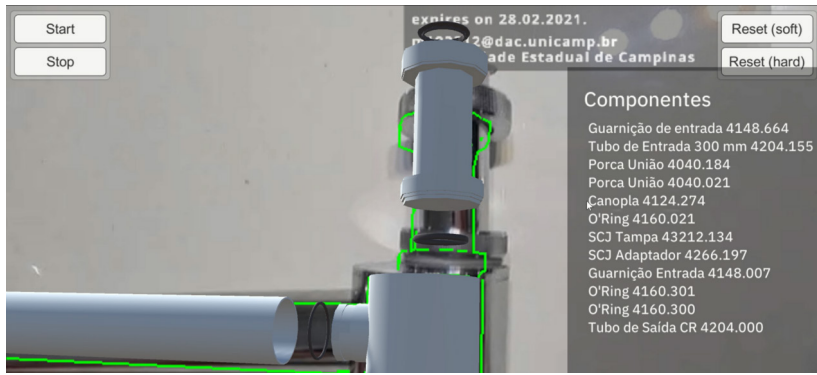

Figure 7. Exploded view superposing the real sink trap

The following four scenes (D, E, F, and G) describe the maintenance instructions provided by the product manufacturer, in summary, respectively: screw the tail pipe into the sink's drain point, measure the distance from the waste pipe to the wall (cut if necessary), connect the trap, and open the inspection cover for maintenance. The four scenes are accompanied by a short animation to illustrate each of these steps. In all moments, the digital model is superimposed on the real sink trap, and it is possible to see the same scene from different angles and distances. The application developed was published for Android devices and Windows platform.

In order to demonstrate the result, the AR application was tested with a washbasin faucet, in which the shape vaguely resembles the sink trap, Figure 8 . At first, the developed application recognizes the faucet shape as part of the sink trap, but as soon as the camera changes the angle, it captures the edges incompatibilities between the real object and the digital model ${ }^{2}$.

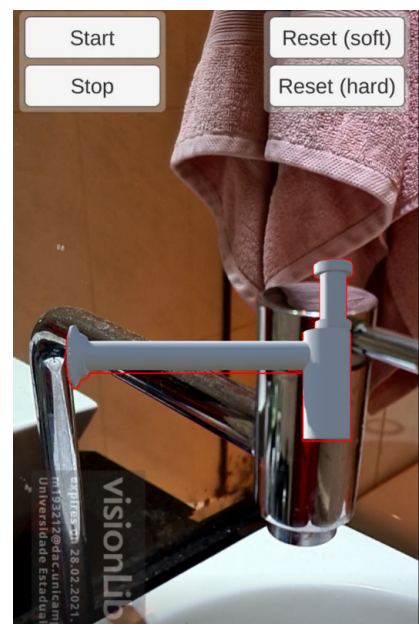

Figure 8. AR application: tracking test

$\overline{2 \text { Video available at: }}$ https://youtu.be/SVd0CtORVXA 


\section{Discussion}

The results obtained are discussed according to its adherence to the initial proposals, and the difficulties and limitations during development are also observed. Among the five requirements, three were fully completed and the others were partially met.

The first requirement (list the sink trap components) has been fully completed. In addition to being possible to list all the components, it was possible to bring extra information for each part, such as its commercial name.

The second requirement (identify/recognize the main components) has been partially completed. The application could successfully recognize the assembled model, relating the digital model to the real model when in AR visualization. However, it was not possible to recognize the three main parts (trap, tail pipe and waste pipe) independently and simultaneously, which would allow the user to detect the sink trap at different stages of its maintenance. For this requirement to be fully met, advanced multi-object tracking feature would be needed and, with it, test whether it would be possible to recognize four combinations: fully disassembled parts, trap uncoupled from the tail and waste pipes, and fully assembled.

The third requirement (warning of rubber seal's position) has also been partially completed. It was possible to show the positioning of the three sealing rubbers on the sink trap from the exploded view, Figure 5 (C). However, the application does not have a warning system, specifying which rubber should be installed and its position. Because of this, it is necessary to retrieve this information from the previous scene.

The fourth requirement (order of components in the assembly) has been fully completed. Scenes (D, E, and F) present the three steps orderly for assembling the sink trap, following the user guide provided by Deca.

The fifth requirement (present the image of the complete sink trap installed) was also completed. So, it was possible to visualize in AR the sink trap assembled and completely disassembled, allowing visualization in advance of the initial and final steps.

Finally, tracking limitations or difficulties are pointed. The main limitation is due to the adaptable dimensions of the cup-shaped trap, which varies depending on the height and distance of the washbasin in each installation, Figure 9.

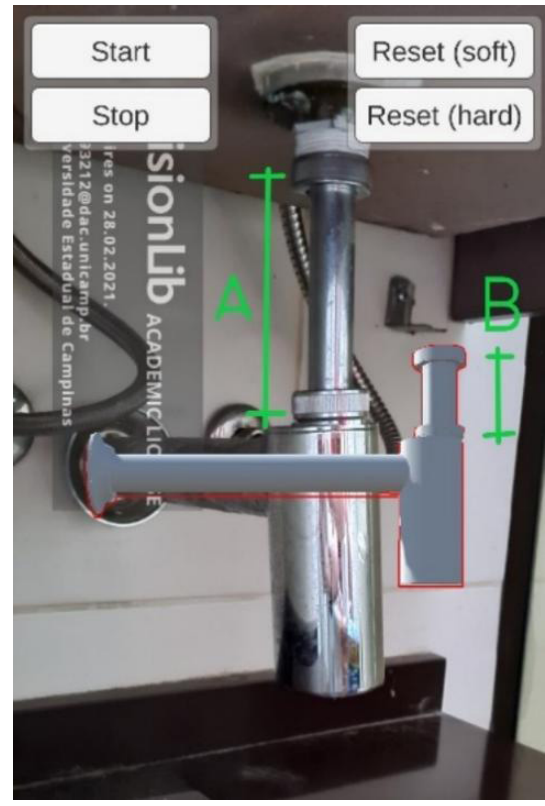

Figure 9. Tracking system and a sink trap with different distances

The second important limitation is the divergence between the model (provided by Deca) and real objects. Between the waste pipe connections to the trap there is an angle, that is not shown the model. The angle between the drainpipe and the trap is not adjustable and the real model has a sloping inclination toward the flow (angle A), while the digital model has a $90^{\circ}$ connection (angle B), Figure 10 .

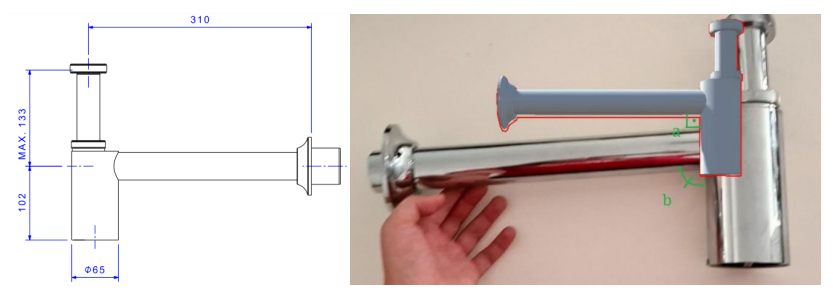

Figure 10. Original model (left) and the real sink trap with different angles (right)

This divergence between angles influenced the model's detection performance by the AR application, which required some attempts to recognize and position the object correctly, but which did not compromise the final tracking ${ }^{1}$.

\section{Conclusions}

In this article, we have presented the results of an AR application developed to assist the maintenance of a cupshaped trap using 3D object recognition. The application was developed based on a sink trap BIM model. A

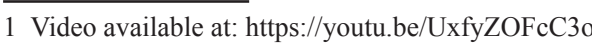


straightforward instruction for the maintenance of the sink trap was provided with animations to lessen work burden and increase efficiency. Although it has been observed that improvements can be made, the application design and results demonstrated the applicability of the system.

Several limitations raised in the literature were also identified in this research: tracking problems (whether by occlusion, dark and tight environments), difficulty in accurately positioning digital models due to parallax errors or due to cylindrical geometry, which is not well defined in all axes (the same view is used for different pipe positions).

Despite these limitations, this study pointed out the feasibility of using AR to aid in the maintenance of piping systems. Specifically, this paper's primary contribution is the investigation of the use of $3 \mathrm{D}$ object tracking to support pipe trap maintenance. Other initiatives may benefit from this study, as it contributes as a practical case and exploratory research on how AR can be accessible to non-specialists to facilitate maintenance, offering an option for visual guidance for facility management tasks.

Future work should focus on WebXR development, as it allows AR content to be accessed by devices such as smartphones, without the need to install applications or plugins. The main advantage of a web application is that it can be run on any system, requiring only a browser to support it.

\section{References}

[1] Milgram P, Takemura H, Utsumi A, Kishino F. Augmented reality: a class of displays on the reality-virtuality continuum. In: Das H, editor. Telemanipulator Telepresence Technol [Internet]. SPIE; 1995. p. 282-92. Available from: https://doi. org/10.1117/12.197321.

[2] Schnabel MA. Framing Mixed Realities. In: Wang X, Schnabel MA, editors. Mix Real Archit Des Constr [Internet]. Dordrecht: Springer Netherlands; 2009. p. 3-11. Available from: https://doi.org/10.1007/978-14020-9088-2_1.

[3] Meža S, Turk Ž, Dolenc M. Measuring the potential of augmented reality in civil engineering. Adv Eng Softw [Internet]. 2015;90:1-10. Available from: https://www.sciencedirect.com/science/article/pii/ S0965997815000915.

[4] Rankohi S, Waugh L. Review and analysis of augmented reality literature for construction industry. Vis Eng. 2013;1:1-18.

[5] Diao PH, Shih NJ. BIM-based AR maintenance system (BARMS) as an intelligent instruction platform for complex plumbing facilities. Appl Sci. $2019 ; 9$.
[6] Pucci A, Conceição D. Estudo Da Incidência De Falhas Visando a Melhoria Da Qualidade Dos Sistemas Prediais Hidráulicos E Sanitários. 2007.

[7] Kwiatek C, Sharif M, Li S, Haas C, Walbridge S. Impact of augmented reality and spatial cognition on assembly in construction. Autom Constr. 2019;108.

[8] Baek F, Ha I, Kim H. Augmented reality system for facility management using image-based indoor localization. Autom Constr [Internet]. Elsevier; 2019;99:18-26. Available from: https://doi. org/10.1016/j.autcon.2018.11.034.

[9] Morikawa K, Ando T. Reduction of piping management person-hours through use of AR technology at shipbuilding sites. Fujitsu Sci Tech J. 2019;55:20-6.

[10] Liu F, Seipel S. Precision study on augmented reality-based visual guidance for facility management tasks. Autom Constr [Internet]. Elsevier; 2018;90:7990. Available from: https://doi.org/10.1016/ j.autcon.2018.02.020.

[11] Han YS, Lee J, Lee J, Lee W, Lee K. 3D CAD data extraction and conversion for application of augmented/virtual reality to the construction of ships and offshore structures. Int J Comput Integr Manuf [Internet]. Taylor \& Francis; 2019;32:65868. Available from: https://doi.org/10.1080/095119 2X.2019.1599440.

[12] Alruwaythi O, Goodrum P. A Difference in Perspective: Impact of Different Formats of Engineering Information and Spatial Cognition on Craft-Worker Eye-Gaze Patterns. J Constr Eng Manag. 2019;145:1-14.

[13] Shen Z, Jiang L. An Augmented 3D iPad Mobile Application for Communication, Collaboration, and Learning (CCL) of Building MEP Systems. Comput Civ Eng [Internet]. 2012. p. 20412. Available from: https://ascelibrary.org/doi/ abs/10.1061/9780784412343.0026.

[14] PTC Inc. Vuforia Developer Library [Internet]. 2021 [cited 2021 May 31]. Available from: https://library. vuforia.com/.

[15] Wikitude GmbH. Wikitude SDK Full Features Overview [Internet]. 2021 [cited 2021 May 31]. Available from: https://www.wikitude.com/products/ wikitude-sdk-features/.

[16] Visometry GmbH. visionLib Docs [Internet]. 2020 [cited 2021 May 31]. Available from: https://docs. visionlib.com/v20.11.1/index.html.

[17] Unity Technologies. Unity - Manual: Unity User Manual 2020.3 (LTS) [Internet]. 2021 [cited 2021 May 31]. Available from: https://docs.unity3d.com/ Manual/index.html. 